\title{
Культурно-исторический феномен гетеротопии в цифровую эпоху
}

\author{
Л.В. Скворцов ${ }^{1}$, Л.Р. Комалова ${ }^{1,2}$, С.С. Сергеев ${ }^{3}$ \\ ${ }^{1}$ Институт научной информации по общественным наукам РАН, \\ ${ }^{2}$ Московский государственный лингвистический университет, \\ ${ }^{3} \mathrm{AHO}$ «Гарант Отношений»
}

Skvortsovcgsir@mail.ru, GenuinePR@yandex.ru, SsSergeev@mai I.ru

\section{Аннотация}

В работе описываются результаты исследования концепции гетеротопии, предложенной Мишелем Фуко, в современной гуманитаристике. Выдвигается гипотеза о том, что гетеротопические пространства в современном мире могут претендовать на роль идеологии в процессе формирования и управления общественным мнением. Общественный дискурс, в свою очередь представляется как инструмент, с помощью которого социальные акторы создают нефизические объекты, в массовом сознании воспринимаемые как реальные. Работа выполнена при поддержке РФФИ, грант № 17-33-00025-ОГН.

Ключевые слова: гетеротопия, иное место, пространство, время, картина мира, дискурс, общественное мнение, воздействие, управление

Библиографическая ссылка: Скворцов Л.В., Комалова Л.Р., Сергеев С.С. Культурно-исторический феномен гетеротопии в цифровую эпоху // Информационное общество: образование, наука, культура и технологии будущего. Выпуск 3 (Труды XXII Международной объединенной научной конференции «Интернет и современное общество», IMS-2019, Санкт-Петербург, 19-22 июня 2019 г. Сборник научных трудов). - СПб: Университет ИТМО, 2019. С. 119 - 127. DOI: $10.17586 / 2587-8557-2019-3-119-127$

\section{1. Введение}

Пятьдесят лет назад Мишель Фуко ввел в научный оборот социально-гуманитарных наук понятие «гетеротопия». За это время данное понятие концептуализировалось применительно к различным сферам исследований. Можно сказать, что гетеротопия стала рабочим понятием современной гуманитаристики. Литературоведы исследуют гетеротопии, воплощаемые авторами художественных произведений в образе «бумажных башен» [1], «чумы» [2], «плантационного хозяйства» [3], «океана» [4], «карнавала» [5], «отеля» [6], читают гетеротопии в «национальном географическом пространстве» [7], «городском пространстве» [8]. Примеры гетеротопических пространств четко просматриваются в кинопродукции [9-12]. С применением концепции гетеротопии исследуются сообщества и административно-территориальные пространства села [13,] и города [14-22]. В условиях геополитических преобразований последнего десятилетия миграционные процессы также рассматриваются как геторотопические объекты [23-26]. Интересным представляется применение концепции гетеротопии в отношении образовательной среды [27-29] и внедрения Интернета и информационных технологий в повседневную жизнь [30-34]. Все чаще авторы рассматривают сетевые ресурсы (facebook, twitter, wikipedia) как цифровые гетеротопии [35]. Авторы утверждаю, что 
гиперпространство и виртуальная реальность являются современными онтологиями, зачастую трансформирующими привычное представление о пространстве и времени [31].

\section{2. Методика исследования}

На стоящей статье представлены результаты мета-анализа научно-исследовательской литературы, в которой, по нашему мнению, представлены описания гетеротопических пространств. В особенности наш интерес привлекают исследования неинституционального дискурса, реализуемого интернет-пользователями в отношении различного рода геополитических преобразований, цивилизационных изменений последних тридцати лет (с момента распада СССР), последовавшие за ними социальные преобразования на национальном и локальном уровнях социального взаимодействия.

В ходе исследования было проанализировано более 200 научных публикаций, извлеченных из международных баз данных научной информации и платформ крупных международных научных издательств: Web of Science, Scopus, elibrary, SieLo, Elsevier, Wiley, SpringerLink, Taylor \& Francis, SAGE, Cambridge University Press, Oxford University Press и др. Предпочтение отдавалось работам за последние десять лет.

\section{3. Результаты}

Результаты анализа геополитического ландшафта позволяют утверждать, что концепция гетеротопии использовалась для присвоения статуса глобального превосходства Соединенными штатами Америки. В этой ситуации статус сверхдержавы обретает та страна, которая обладает моральным превосходством, позволяющим остановить опасность извержения «термоядерного» вулкана, избежать человечеству превращения в «ничто».

C позиций гетеротопического анализа геополитические конфликты представляют наибольший интерес в силу того, что изменениям подвергаются цивилизационные контуры в глобальном масштабе. В связи с этим возрастает необходимость учета социальных контекстов геополитического конфликта в целях возможной институционализации конфликта как поиска новых форм социальных отношений, в которых в кооперативной форме протекала бы эскалация и урегулирование конфликтных взаимодействий. Конфронтационный и консенсуальный подходы к анализу конфликтного взаимодействия рассматриваются как необходимая полярная пара при превентивных формах интервенции в геополитические конфликты и их «лечения».

В геополитических конфликтах возникает и начинает одновременно действовать множество конфликтогенных факторов, приводящих к хаотической эскалации, что начинает наделять все разнообразие конфликтных взаимодействий свойствами гетеротопного пространства. К базовым механизмам эскалации социальных конфликтов можно отнести следующие:

- конфликтующие партии склонны проецировать и приписывать негативные установки и деструктивное поведения представителям противоположной стороны, часто стремясь создать образ врага;

- конфликтующие партии склонны редуцировать сложность возникающей конфликтной ситуации, при этом расширяя число и глубину спорных вопросов;

- конфликтующие партии склонных постоянно втягивать в конфликт для обеспечения поддержки общественные объединения и третьих лиц и формировать мнение в интересах собственных позиций;

- конфликтующие стороны склонны использовать угрозы применения силовых методов с целью склонить противоположную сторону к уступкам, что, однако, приводит к ответным угрозам и усилению эскалации. 
При этом эскалационные процессы в конфликте имеют ступенчатую организацию и по мере перехода от одной фазы эскалации геополитического конфликта к другой происходит регрессия, и конфликтующие партии теряют целый комплекс возможностей по урегулированию конфликта и исключаются многие альтернативы.

Концепция гетеротопии представляется в равной мере аппликабельной как на мировом, так и на региональном уровнях взаимодействия исследуемых субъектов. В качестве частного случая гетеротопии на локальном уровне можно рассматривать «пространство протеста» (по М. Фуко), в котором гражданские движения выстраивают «иное пространство» принятия политических решений в противовес легитимной власти в виде политических партий.

\section{4. Заключение}

Проведенный анализ научной литературы показывает, что было бы ошибочно упрощать эпистемологическое толкование феномена гетеротопии как фантомной реальности. Напротив, в цифровую эру гетеротопии обретают более чем реальные очертания. Феномен гетеротопии имеет фундаментальное значение для легитимации принципиально нового методологического подхода к интерпретации исторического цивилизационного процесса. Речь идет о переоценке конечных целей цивилизационной эволюции. Концепция гетеротопии отвергает идею единого закономерного восхождения цивилизационной эволюции к своей конечной цели [36]; рассматривает историческую реальность как сумму различных цивилизационных мест, множественности страт, кратких очередностей, восстающих против единого закона.

Концепция гетеротопии может также рассматриваться как оправдание отношения к построению жизни как такой игре, которая создает для человека, осознавшего реальность современной глобальной угрозы для жизни, собственный магический круг бытия, пространственной обособленности, убежище, позволяющее сохранить себя в ином, фантастическом мире. Такой подход отрывает возможности для перелицовки реалий жизни и исторических фактов, для замены «сокровенных сокровищ вещей» дискурсом, регулярной формацией объектов. Установление такого рода объектов дискурса не требует исследования их действительного смысла, поскольку сама эпоха характеризовала этот смысл как «тихое помешательство» [37]. Такой дискурс не отсылает к идеальной логике или эмпирическому генезису понятий и может существовать на доконцептуальном уровне как действие теоретических схем типа атрибуции, артикуляции, обозначения и деривации. Эти схемы определяют область правомерности критериев диспутирования об истинности и ложности. Этот уровень выводов не отсылает к идеальной логике и эмпирическому генезису абстракций. Таким образом, дискурс не является адекватным отображением реальности. Он является местом рождения концептов. Однако именно дискурсивное пространство создает иную реальность, которая при наличии общего согласия может претендовать на статус признанной истины, в отношении которой истина, опирающаяся на реальность общей первоначальной почвы, может подвергаться скептическому отрицанию как субъективная точка зрения.

Методология формирования дискурсивных объектов оказалась пригодной для создания системы управления общественным мнением современного мира: глобальное управление общественным мнением приобретает приоритетный смысл. Это форма информационной диктатуры, опирающаяся на владение средствами информации, теоретические разработки и авторитет согласованной коллективной позиции. Так дискурсивные объекты становятся основанием «правильного» и «неправильного», независимых от сущности объективной истины. Дискурсивная объективность становится очевидной реальностью в формах массового поведения, а значит и в его результатах как продуктах этого поведения. Таким образом, дискурсивные объективности в своих идеальных формах начинают соответствовать создаваемой реальности жизни. Таким образом, считает М. Фуко, 
освобождаются совокупности правил, обусловливающих дискурсивную практику. Так формируется «поле присутствия», то, что попадает в поле наблюдения, передается из уст в уста, что можно определять как память. Так количественные высказывания могут переводиться в качественные, т.е. формы иерархии и подчинения, комментирования и интерпретации различных точек зрения.

Противоречия XX века исключили возможность обнаружения места бытия свободного от угрозы социальных и военных столкновений [38]. Выдвинутая М. Фуко концепция гетеротопии как места смысла, находящегося между реальным бытием человека и утопией идеального бытия, условно позволяет «вырваться» из замкнутого круга. Однако, оказываясь между смысловыми слоями бытия, человек становится объектом информационного манипулирования субъектов, способных созидать частные гетеротопии. Сущность гетеротопии раскрывает дискурсивная объективность, коллективно признаваемая как действительность, воспринимаемая массовым сознанием в качестве истины.

В современном обществе концепция М. Фуко объективно претендует на замещение религиозных и идеологических форм определения исходных принципов миропонимания, массового поведения и практики жизни. В связи с этим М. Фуко можно считать автором концепции идеологии нового типа, создающей словесный порядок, рождающий собственный объект, понимаемый как последовательность словесных знаков, воспринимаемая как овеществленная идеология.

Принимая во внимание шесть принципов (условий существования) гетеротопии можно утверждать, что гетеротопия как семиотическая система может быть представлена как концентрированный вариант мировоззрения современного человека, находящегося в постоянном поиске, построении и разрушении собственного места в пространстве социального.

В целом можно утверждать, что концепция гетеротопии применима в отношении исследования следующих проблемных областей: вопросы современных информационных взаимодействий; исследования достоверности информационного освещения действительности; проблемы разрешения современных конфликтных ситуаций; проблемы взаимодействия политических партий и общественных движений; литературная интерпретация отношения героя к действительности; философская интерпретация гетеротопии как формы объяснения встречи личного «Я» с неопределенностью бытия.

Работа выполнена при поддержке Российского фонда фундаментальных исследований, грант № 17-33-00025-ОГН «Гетеротопия: Цивилизационный контекст».

\section{Литература}

[1] Wilkinson L. Nerdfighters, «Paper Towns», and heterotopia // Transformative Works and Cultures. 2012. Vol. 10. DOI: https://doi.org/10.3983/twc.2012.0374. https://journal.transformativeworks.org/index.php/twc/article/view/374/301.

[2] Хрящева Н.П., Когут К.С. Гетеротопия чумы в романе А.В. Иванова «Комьюнити» // Филологический класс. 2013. № 3 (33). С. 33 - 40.

[3] Якушенков С.Н., Самсонова Н.В. Проблема сохранения культурной безопасности в условиях гетеротопных пространств фронтира (На примере Астраханской области) // Каспийский регион: политика, экономика, культура. 2013. № 4. С. 232 - 238.

[4] Дырдин А.А. Гетеротопология как стратегия литературно-философских исследований // Филологический класс. 2015. № 4. С. 18 - 22.

[5] Quigley G. Burroughs and his world: Carnival as heterotopia in Naked Lunch // NEW ACADEMIA: An International Journal of English Language, Literature and Literary Theory. 2015. Vol. IV. Issue II, P. 80-88. https://interactionsforum.com/images/pdfs/ newacademia/v4/i2/nine-9.pdf. 
[6] Martínez M.L.M., Torres P.G. Bad days will come for love and forgetting: The place of memory in Miramar Hotel by Egor Mardones // Atenea. 2015. Vol. 512. P. 269.

[7] Beebee Th.O. Geographies of nation and region in modern European and American fiction // CLCWeb: Comparative Literature and Culture. 2008. Vol. 10, Issue 3. DOI: https://doi.org/10.7771/1481-4374.1377. https://docs.lib.purdue.edu/clcweb/vol10/iss3/7/.

[8] Boettcher N. Identity formation in Paul Auster's fictional urban space // Romanian Journal of English Studies. 2013. Vol. 10, Issue 1. P. 221 - 227. DOI: https://doi.org/10.2478/rjes2013-0020. https://content.sciendo.com/view/journals/rjes/10/1/article-p221.xml.

[9] Blair L.S. Driving toward heterotopias: Taxis and taxistas in contemporary Chilean cinema // Letras Hispanas. 2012. Vol. 8.1. P. 21-31. https://gato-docs.its.txstate.edu/jcr:87b7b1b148b2-48d7-940a-d6e15715240c/L.S.\%20Blair.pdf.

[10]Filimon E.C. Heterotopia on Screen-Blue Velvet (1986) // Romanian Journal of English Studies. 2014. Vol. 11. Issue 1. P. 158 - 166. DOI: https://doi.org/10.2478/rjes-2014-0020. https://content.sciendo.com/view/journals/rjes/11/1/article-p158.xml.

[11] Puric B. Matka, a heterotopia of deviation // Short Film Studies: Intellect. 2014. Vol. 4, № 1. P. $43-46$.

[12] Ersöz Koç E. Control and resistance in the heterotopic spatiality of Pleasantville // CINEJ Cinema Journal. 2015. Vol. 5. Issue 1. P. 58 - 83. DOI: https://doi.org/10.5195/cinej.2015.125. http://cinej.pitt.edu/ojs/index.php/cinej/article/view/125/364.

[13] Алиев Р.Т. Совет старейшин как социокультурный элемент в гетеротопном пространстве Астраханского фронтира (На примере астраханских татар села Осыпной бугор) // Каспийский регион: политика, экономика, культура. 2015. № 4 (45). С. 315 - 317.

[14]Квят А.Г. «Все будет иначе»: Городской пикник как гетеротопия // Вестник Томского государственного университета. 2014. № 388. С. 65 - 75.

[15]Рыжкова Д.С. Городские пространства: От утопии к гетеротопии // Актуальные проблемы гуманитарных и естественных наук. 2014. № 1-1. С. 233 - 237.

[16]Хлыщева Е.В. Свой и чужой в гетеротопном пространстве медиа-культуры // Каспийский регион: политика, экономика, культура. 2015. № 3 (44). С. 275 - 280.

[17]Гутыра В.И. Феномен деревни Окунево: Субкультурное пространство городской молодежи // Журнал института наследия. 2016. № 1 (4). http://naslediejournal.ru/ru/journals/57.html.

[18] Rossetto M. Heterotopia and its role in the lived experiences of resettlement // International Education Journal. 2006. Vol. 7. Issue 4. P. 446 - 454. https://eric.ed.gov/?id=EJ854300.

[19] Silva Echeto V., Sartori R.B. Las ciudades invisibles: heterotopías nómadas y postpatriarcado // Revista Estudos Feministas. 2009. Vol. 17. Issue 2. P. 335 - 348. DOI: https://doi.org/10.1590/S0104-026X2009000200003.

https://periodicos.ufsc.br/index.php/ref/article/view/S0104-026X2009000200003.

[20] Sudradjat I. Foucault, the Other Spaces, and human behaviour // Procedia - Social and Behavioral Sciences. 2012. Vol. 36. P. 28 - 34. DOI: 10.1016/j.sbspro.2012.03.004

[21] Hassan R. Decoding the 'City': A study of the Barn Owl's Wondrous Capers // International Journal of Humanities and Social Studies. 2012. Vol 2. No 12.

[22] Kassens-Noor E. From ephemeral planning to permanent urbanism: An urban planning theory of mega-events // Urban Planning. 2016. Vol. 1. Issue 1. P. 41 - 54. DOI: http://dx.doi.org/10.17645/up.v1i1.532. https://www.cogitatiopress.com/urbanplanning/article/ view/532.

[23]Рителла Д. Присутствие, социальное присутствие и гетеротопия: Я и другие в мультипространстве // Культурно-историческая психология. 2010. № 4. С. 39 - 46.

[24]Лебедева И.В. Политика мультикультурализма и безопасность в Европе // Современная наука и инновации. 2015. № 2 (10). С. 156 - 161. 
[25]Хлыщева Е.В. Проблемы культурного взаимодействия в условиях мультикультурной гетеротопии // Каспийский регион: политика, экономика, культура. 2015. № 4 (45). С. $260-264$.

[26]Баева Л.В. Современные миграционные процессы в контексте теории гетеротопии // Каспийский регион: политика, экономика, культура. 2016. № 3 (48). С. 100 - 106.

[27] Gosling D. Teaching and learning projects as 'heterotopias' // Critical Studies in Teaching and Learning. 2014. Vol. 2. Issue 1. P. 25 - 48. DOI: http://dx.doi.org/10.14426/cristal.v2i1.18.

https://www.ajol.info/index.php/cristal/article/view/125964.

[28] Sandberg F., Fejes A., Dahlstedt M., Olson M. Adult education as a heterotopia of deviation: A dwelling for the abnormal citizen // Adult Education Quarterly. 2015. Vol. 66. Issue 2. P. $103-119$.

[29] Gourlay L. Open education as a «heterotopia of desire» // Learning, Media and Technology. 2015. Vol. 40, Issue 3: Critical Approaches to Open Education. P. 310 - 327. DOI: 10.1080/17439884.2015.1052622.

https://www.tandfonline.com/doi/full/10.1080/17439884.2015.1052622.

[30] Thielmann T. "You have reached your destination!» Position, positioning and superpositioning of space through car navigation systems // Social Geography. 2007. Vol. 2. P. 63 - 75. DOI: 10.5194/sg-2-63-2007. http://www.soc-geogr.net/2/63/2007/sg-2-632007.html.

[31]Pertierra R. The new media and heterotopic technologies in the Philippines. Human Technology: An Interdisciplinary // Journal on Humans in ICT Environments. 2008. Vol. 4 (2). P. 169 - 185. https://jyx.jyu.fi/dspace/handle/123456789/20226.

[32] Haider J., Sundin O. Beyond the legacy of the enlightenment? Online encyclopaedias as digital heterotopias // First Monday. 2010. Vol. 15. № 1-4. http://journals.uic.edu/ojs/index.php/fm/article/view/2744/2428.

[33] Anderson J., Bharthapudi K., Cao H. Occupy the heterotopia // International Review of Information Ethics. 2012. Vol. 18. P. 152 - 168. http://www.i-r-i-e.net/inhalt/018/AndersonBharthapudi-Cao.pdf.

[34] Rymarczuk R., Derksen M. Different spaces: Exploring Facebook as heterotopia // First Monday. 2014. Vol. 19. № 6-2. http://journals.uic.edu/ojs/index.php/fm/article/ view/5006/4091.

[35] Komalova L. Social network sites as digital heterotopias: Textual content and speech behavior perception // Alexandrov D., Boukhanovsky A., Chugunov A., Kabanov Y., Koltsova O. (eds.): Digital transformation and global society. DTGS 2018, Communications in Computer and Information Science. 2018. Vol. 859. P. 43 - 54. Communications in Computer and Information Science series. DOI: https://doi.org/10.1007/978-3-030-02846-6_4.

[36] Скворцов Л.В. Функции информации в межцивилизационных отношениях: К оценке концепции гетеротопии // Россия: тенденции и перспективы развития. М.: ИНИОН PAH, 2017a. Вып. 12. Ч. 3. С. 834-835. http://inion.ru/publishing/publications/rossiiatendentsii-i-perspektivy-razvitiia-ezhegodnik-12-3/.

[37]Скворцов Л.В. Истина гетеротопии: Между фантомом и реальностью. Часть 2 // Человек: Образ и сущность. Гуманитарные аспекты = Human being: Image and essence. Humanitarian aspects. М.: ИНИОН РАН, 2017б. № 3-4 (30-31): Угроза апокалипсиса и идея сверхчеловека $=$ The threat of the apocalypse and the idea of the superman. C. 13-23. https://elibrary.ru/item.asp?id=32402088.

[38]Скворцов Л.В. Понятие гетеротопии и современный мировой порядок: К постановке проблемы // Россия и современный мир. М.: ИНИОН РАН, 2018. № 1. С. 219-232. DOI: 10.31249/rsm/2018.01.15. http://rossovmir.ru/article.php?id=243. 


\title{
Cultural and Historical Phenomenon of Heterotopia in Digital Era
}

\author{
L.V. Skvortsov ${ }^{1}$, L.R. Komalova ${ }^{1,2}$, S.S. Sergeev ${ }^{3}$ \\ ${ }^{1}$ Institute of Scientific Information for Social Sciences of the Russian Academy of Sciences, \\ ${ }^{2}$ Moscow State Linguistic University, \\ ${ }^{3}$ Autonomous non-commercial organization «Guarantor of Relations»
}

The paper explores the concept of heterotopy, proposed by Michel Foucault, in modern Humanitarian Sciences. It is hypothesized that heterotopic spaces in the modern world can claim to be an ideology in the process of forming and managing public opinion. Public discourses, in turn, are presented as a means with which social actors create non-physical objects that are perceived as real in the public consciousness. The reported study was funded by the Russian Foundation for Basic Researches according to the research project № 17-33-00025-ОГН.

Keywords: heterotopy, other place, space, time, image of the world, discourse, public opinion, impact, management

Reference for citation: Skvortsov L.V., Komalova L.R., Sergeev S.S. Cultural and Historical Phenomenon of Heterotopia in Digital Era // Information Society: Education, Science, Culture and Technologies of the Future. Vol. 3 (Proceedings of the XXII International Joint Scientific Conference «Internet and Modern Society», IMS-2019, St. Petersburg, June 19-22, 2019). St. Petersburg: ITMO University, 2019. P. 119 - 127. DOI: 10.17586/2587-8557-2019-3-119-127

\section{Reference}

[1] Wilkinson L. Nerdfighters, «Paper Towns», and heterotopia // Transformative Works and Cultures. 2012. Vol. 10. DOI: https://doi.org/10.3983/twc.2012.0374. https://journal.transformativeworks.org/index.php/twc/article/view/374/301.

[2] Hryashcheva N.P., Kogut K.S. Geterotopiya chumy v romane A.V. Ivanova «Kom'yuniti» // Filologicheskij klass. 2013. № 3 (33). P. 33 - 40.

[3] Yakushenkov S.N., Samsonova N.V. Problema sohraneniya kul'turnoj bezopasnosti v usloviyah geterotopnyh prostranstv frontira (Na primere Astrahanskoj oblasti) // Kaspijskij region: politika, ekonomika, kul'tura. 2013. № 4. P. 232 - 238.

[4] Dyrdin A.A. Geterotopologiya kak strategiya literaturno-filosofskih issledovanij // Filologicheskij klass. 2015. № 4. P. $18-22$.

[5] Quigley G. Burroughs and his world: Carnival as heterotopia in Naked Lunch // NEW ACADEMIA: An International Journal of English Language, Literature and Literary Theory. Vol. IV. Issue II, 2015. P. 80-88. https://interactionsforum.com/images/pdfs/newacademia/ v4/i2/nine-9.pdf.

[6] Martínez M.L.M., Torres P.G. Bad days will come for love and forgetting: The place of memory in Miramar Hotel by Egor Mardones // Atenea. 2015. Vol. 512. P. 269.

[7] Beebee Th.O. Geographies of nation and region in modern European and American fiction // CLCWeb: Comparative Literature and Culture. 2008. Vol. 10, Issue 3. DOI: https://doi.org/10.7771/1481-4374.1377. https://docs.lib.purdue.edu/clcweb/vol10/iss3/7/.

[8] Boettcher N. Identity formation in Paul Auster's fictional urban space // Romanian Journal of English Studies. 2013. Vol. 10, Issue 1. P. 221 - 227. DOI: https://doi.org/10.2478/rjes2013-0020. https://content.sciendo.com/view/journals/rjes/10/1/article-p221.xml.

[9] Blair L.S. Driving toward heterotopias: Taxis and taxistas in contemporary Chilean cinema // Letras Hispanas. 2012. Vol. 8.1. P. 21-31. https:/gato-docs.its.txstate.edu/jcr:87b7b1b148b2-48d7-940a-d6e15715240c/L.S.\%20Blair.pdf. 
[10]Filimon E.C. Heterotopia on Screen-Blue Velvet (1986) // Romanian Journal of English Studies. 2014. Vol. 11. Issue 1. P. 158 - 166. DOI: https://doi.org/10.2478/rjes-2014-0020. https://content.sciendo.com/view/journals/rjes/11/1/article-p158.xml.

[11] Puric B. Matka, a heterotopia of deviation // Short Film Studies: Intellect. 2014. Vol. 4. N. 1. P. $43-46$.

[12] Ersöz Koç E. Control and resistance in the heterotopic spatiality of Pleasantville // CINEJ Cinema Journal. 2015. Vol. 5. Issue 1. P. $58-83$. DOI: https://doi.org/10.5195/cinej.2015.125. http://cinej.pitt.edu/ojs/index.php/cinej/article/view/125/364.

[13] Aliev R.T. Sovet starejshin kak sociokul'turnyj element $\mathrm{v}$ geterotopnom prostranstve Astrahanskogo frontira ( $\mathrm{Na}$ primere astrahanskih tatar sela Osypnoj bugor) // Kaspijskij region: politika, ekonomika, kul'tura. 2015. № 4 (45). P. 315 - 317.

[14] Kvyat A.G. «Vse budet inache»: Gorodskoj piknik kak geterotopiya // Vestnik Tomskogo gosudarstvennogo universiteta. 2014. № 388. P. 65 - 75 .

[15]Ryzhkova D.S. Gorodskie prostranstva: Ot utopii k geterotopii // Aktual'nye problemy gumanitarnyh i estestvennyh nauk. 2014. № 1-1. P. 233 - 237.

[16]Hlyshcheva E.V. Svoj i chuzhoj v geterotopnom prostranstve media-kul'tury // Kaspijskij region: politika, ekonomika, kul'tura. 2015. № 3 (44). P. 275 - 280.

[17] Gutyra V.I. Fenomen derevni Okunevo: Subkul'tunoe prostranstvo gorodskoj molodezhi // ZHurnal instituta naslediya. 2016. № 1 (4). http://nasledie-journal.ru/ru/journals/57.html.

[18] Rossetto M. Heterotopia and its role in the lived experiences of resettlement // International Education Journal. 2006. Vol. 7. Issue 4. P. 446 - 454. https://eric.ed.gov/?id=EJ854300.

[19] Silva Echeto V., Sartori R.B. Las ciudades invisibles: heterotopías nómadas y postpatriarcado // Revista Estudos Feministas. 2009. Vol. 17. Issue 2. P. 335 - 348. DOI: https://doi.org/10.1590/S0104-026X2009000200003. https://periodicos.ufsc.br/index.php/ref/article/view/S0104-026X2009000200003.

[20] Sudradjat I. Foucault, the Other Spaces, and human behaviour // Procedia - Social and Behavioral Sciences. 2012. Vol. 36. P. 28 - 34. DOI: 10.1016/j.sbspro.2012.03.004.

[21] Hassan R. Decoding the 'City': A study of the Barn Owl's Wondrous Capers // International Journal of Humanities and Social Studies. 2012. Vol 2. No 12..

[22] Kassens-Noor E. From ephemeral planning to permanent urbanism: An urban planning theory of mega-events // Urban Planning. 2016. Vol. 1. Issue 1. P. 41 - 54. DOI: http://dx.doi.org/10.17645/up.v1i1.532.

https://www.cogitatiopress.com/urbanplanning/article/view/532.

[23] Ritella D. Prisutstvie, social'noe prisutstvie i geterotopiya: YA i drugie v mul'tiprostranstve // Kul'turno-istoricheskaya psihologiya. 2010. № 4. P. 39 - 46.

[24] Lebedeva I.V. Politika mul'tikul'turalizma i bezopasnost' v Evrope // Sovremennaya nauka i innovacii. 2015. № 2 (10). P. 156 - 161.

[25]Hlyshcheva E.V. Problemy kul'turnogo vzaimodejstviya v usloviyah mul'tikul'turnoj geterotopii // Kaspijskij region: politika, ekonomika, kul'tura. 2015. № 4 (45). P. 260 - 264.

[26] Baeva L.V. Sovremennye migracionnye processy v kontekste teorii geterotopii // Kaspijskij region: politika, ekonomika, kul'tura. 2016. № 3 (48). P. 100 - 106.

[27] Gosling D. Teaching and learning projects as 'heterotopias' // Critical Studies in Teaching and Learning. 2014. Vol. 2. Issue 1. P. 25 - 48. DOI: http://dx.doi.org/10.14426/cristal.v2i1.18. https://www.ajol.info/index.php/cristal/article/view/125964.

[28] Sandberg F., Fejes A., Dahlstedt M., Olson M. Adult education as a heterotopia of deviation: A dwelling for the abnormal citizen // Adult Education Quarterly. 2015. Vol. 66. Issue 2. P. $103-119$. 
[29] Gourlay L. Open education as a «heterotopia of desire» // Learning, Media and Technology. 2015. Vol. 40, Issue 3: Critical Approaches to Open Education. P. 310 - 327. DOI: 10.1080/17439884.2015.1052622. https://www.tandfonline.com/doi/full/10.1080/17439884.2015.1052622.

[30] Thielmann T. "You have reached your destination!» Position, positioning and superpositioning of space through car navigation systems // Social Geography. 2007. Vol. 2. P. 63 - 75. DOI: 10.5194/sg-2-63-2007. http://www.soc-geogr.net/2/63/2007/sg-2-632007.html.

[31]Pertierra R. The new media and heterotopic technologies in the Philippines. Human Technology: An Interdisciplinary Journal on Humans in ICT Environments. 2008. Vol. 4 (2). P. 169 - 185. https://jyx.jyu.fi/dspace/handle/123456789/20226.

[32] Haider J., Sundin O. Beyond the legacy of the enlightenment? Online encyclopaedias as digital heterotopias // First Monday. 2010. Vol. 15. № 1-4. http://journals.uic.edu/ojs/index.php/fm/article/view/2744/2428.

[33] Anderson J., Bharthapudi K., Cao H. Occupy the heterotopia // International Review of Information Ethics. 2012. Vol. 18. P. 152 - 168. http://www.i-r-i-e.net/inhalt/018/AndersonBharthapudi-Cao.pdf.

[34]Rymarczuk R., Derksen M. Different spaces: Exploring Facebook as heterotopia // First Monday. $2014 . \quad$ Vol. $19 . \quad$ № 6-2. http://journals.uic.edu/ojs/index.php/fm/article/view/5006/4091.

[35] Komalova L. Social network sites as digital heterotopias: Textual content and speech behavior perception // Alexandrov D., Boukhanovsky A., Chugunov A., Kabanov Y., Koltsova O. (eds.): Digital transformation and global society. DTGS 2018, Communications in Computer and Information Science. 2018. Vol. 859. P. 43 - 54. Communications in Computer and Information Science series. DOI: https://doi.org/10.1007/978-3-030-0284664.

[36] Skvortsov L.V. Funkcii informacii v mezhcivilizacionnyh otnosheniyah: K ocenke koncepcii geterotopii // Rossiya: tendencii i perspektivy razvitiya. M.: INION RAN, 2017a. Issue 12. Part 3. P. 834-835. http://inion.ru/publishing/publications/rossiia-tendentsii-i-perspektivyrazvitiia-ezhegodnik-12-3/.

[37] Skvortsov L.V. Truth of heterotopia: Between phantom and reality. Part 2 // Human Being: Image and Essence. Humanitarian Aspects. 2017b. № 3-4 (30-31): The threat of the apocalypse and the idea of the superman. P. 13-23. https://elibrary.ru/item.asp?id=32402088.

[38] Skvortsov L.V. The concept of heterotopia and the contemporary world order: The problem statement // Russia and the Contemporary World. 2018. № 1. P. 219-232. DOI: 10.31249/rsm/2018.01.15. http://rossovmir.ru/en/article.php?id=243. 